\title{
Equation-of-state behavior for different phases of lead under strong compression
}

\author{
Olaf Schulte and Wilfried B. Holzapfel \\ Universität Gesamthochschule Paderborn, 33095 Paderborn, Germany
}

(Received 20 June 1995)

\begin{abstract}
X-ray diffraction data for lead under pressures up to $80 \mathrm{GPa}$ are used together with previous data covering a wider range to determine the equations of state (EOS) for the three different phases which occur under these pressures at ambient temperature. It is shown that the first-order EOS form $H 11$, typical for "simple" solids, describes perfectly all the data when only the values for $V_{0}$ and $K_{0}$ are individually adapted for each phase.
\end{abstract}

\section{INTRODUCTION}

The progress in high-pressure physics due to the ability to study small sample volumes connected with powerful x-ray sources allows to investigate properties of solids in a static pressure range up to $300 \mathrm{GPa}$ and above. ${ }^{1}$ Pressure-volume data at those pressures have been obtained before only by shock-wave experiments; however, no structural information is available from this method and critical assumptions have to be made in the evaluation of isotherms from the Hugoniot data. ${ }^{2}$ With the rapid extension of the pressure range divergences of different equation-of-state (EOS) forms with respect to the limiting behavior of a free-electron gas become obvious, however, these deficiencies can be avoided by recently derived EOS forms as shown in various recent applications. ${ }^{3-9}$ These studies resulted in a classification of materials into three groups with respect to their highpressure behavior: "ideal" solids; their high-pressure behavior can be described accurately with a "zeroth order" EOS form using the zero-pressure volume $V_{0}$ and an average atomic number $Z$ as the only input parameters. "Simple" solids are thereby characterized by one additional parameter, typically $K_{0}$, the bulk modulus at ambient conditions, which determines all the other higher-order pressure derivatives like $K_{0}^{\prime}, K_{0}^{\prime \prime}$ and so on. Second- or higher-order forms are finally needed for all the other solids with nonsimple behavior, where $Z, V_{0}, K_{0}$ and sometimes even higher-order parameters may enter into an accurate representation of the corresponding EOS data. This last group contains most materials with phase transitions under pressure. To describe the behavior of these materials under strong compression, each phase must be represented by its individual EOS.

From this point of view, one may consider lead at first as an element with nonsimple behavior, as it shows two phase transitions under pressure with small volume discontinuities. Initially, a continuous pressure-volume relation was determined up to $1000 \mathrm{GPa}$ by shock-wave experiments, ${ }^{10}$ however, later phase transitions and the corresponding volume discontinuities were found by energy-dispersive x-ray diffraction under pressures up to $272 \mathrm{GPa}$ (Refs. 11 and 12). Furthermore, there are some pseudopotential calculations, which reproduce the high-pressure behavior of lead in good agreement with the experimental results. ${ }^{10,13}$

In this paper, additional x-ray diffraction data for lead up to $80 \mathrm{GPa}$ are presented for the intermediate region to allow for a more accurate distinction of the EOS forms for the different phases. Together with data of lead from the literature, the parameters $K_{0}$ and $K_{0}^{\prime}$ as well as $V_{0}$ for the highpressure phases are evaluated by fitting appropriate EOS forms to the data in order to make a comparison with previous results.

\section{EXPERIMENTAL}

Diffraction patterns of lead are obtained by energydispersive $\mathrm{x}$-ray diffraction using either a conventional $\mathrm{x}$-ray tube together with the conical slit arrangement ${ }^{14}$ or synchrontron radiation with a standard diffraction setup ${ }^{15}$ at the EDS station F3 of HASYLAB, DESY, with its recent modifications. ${ }^{16}$ Pressures were generated by a gasketed diamond-anvil cell ${ }^{17,18}$ with beveled diamonds and measured with the ruby-luminescence technique ${ }^{19}$ using the nonlinear pressure scale. ${ }^{20}$ To maintain quasihydrostatic conditions, mineral oil was used as pressure transmitting medium.

\section{RESULTS AND DISCUSSION}

Diffraction patterns of lead were measured up to $80 \mathrm{GPa}$ confirming first of all the phase transition from the lowpressure $c F 4$ phase to the intermediate $h P 2$ phase of lead around $14 \mathrm{GPa}$ (here and throughout this paper the structural nomenclature recommended by IUPAC is used ${ }^{21}$ ). The effect

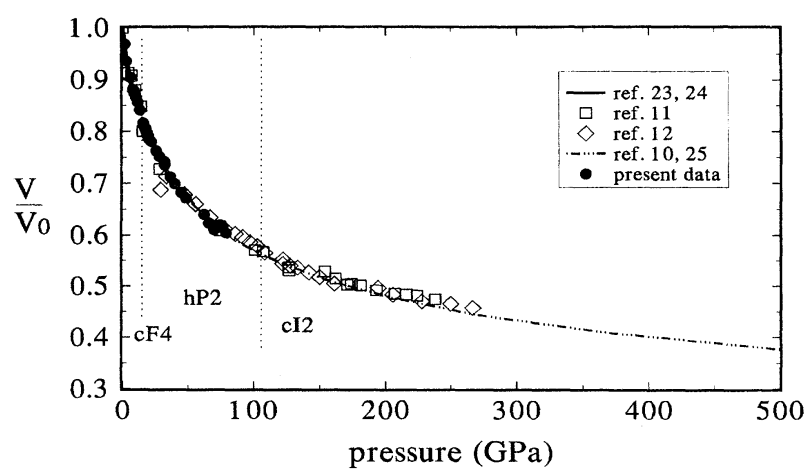

FIG. 1. Pressure-volume data for the three different phases of lead at ambient temperature using $V_{0}=30.33 \times 10^{6} \mathrm{pm}^{3}$ (Ref. 22). 
of pressure on the atomic volume is illustrated for the range from 0 to $500 \mathrm{GPa}$ in Fig. 1 which includes in addition to the present data previous results from volumetric, ${ }^{23,24}$ $\mathrm{x}$-ray-diffraction, ${ }^{11,12}$ and shock-wave ${ }^{10,25}$ measurements. Obviously, this form of representation for the different data sets does not bring out the differences in the EOS forms for the different phases.

These differences can be seen, however, if one uses a special "linearization scheme" ${ }^{4}$ for the data as illustrated in Fig. 2 with

$$
\eta=\ln \left[\frac{p X^{5}}{p_{\mathrm{FG} 0}}\right]-\ln (1-X),
$$

where by $X=\sigma / \sigma_{0}$, and $\sigma=(3 Z V / 4 \pi)^{1 / 3}$ represents the Wigner-Seitz-radius scaled by the third root of the atomic number $Z, p_{\mathrm{FG}}=a_{\mathrm{FG}}(Z / V)^{5 / 3}$ is the Fermi-gas pressure with the universal constant $a_{\mathrm{FG}}=23.369 \mathrm{MPa} \mathrm{nm}^{5}$, and the subscript 0 is used for ambient pressure values. First of all, this representation shows for the low-pressure $c F 4$ phase small but significant differences between the different data sets especially in comparison with the best value for the isothermal bulk modulus $K_{0}$ from ultrasonic (US) measurements. ${ }^{26}$ The continuous curve represents a linear interpolation between the starting value $\eta_{0}=\ln \left(3 K_{0} / p_{\mathrm{FG} 0}\right)$ using the best value for $K_{0}$ from the US data, and the ultimate value $\eta \rightarrow 0$ for $X \rightarrow 0$. The EOS form corresponding to this linear interpolation has been called $H 11$ (Refs. 3 and 4) and has the form

$$
p=3 K_{0} X^{-5}(1-X) \exp \left[c_{0}(1-X)\right]
$$

with $c_{0}=-\ln \left(3 K_{0} / p_{\mathrm{FG} 0}\right)$ and $K_{0}^{\prime}=3+2 c_{0} / 3$. While the previous $\mathrm{X}$-ray data deviate in opposite directions from the present data, good agreement with the $H 11$ curve is found with respect to the volumetric data and for the (smoothed) shock-wave results. These differences are also seen in the EOS parameters in Table I where close agreements are found for $K_{0}$ and $K_{0}^{\prime}$ just in the later cases.

For the high-pressure phases $h P 2$ and $c I 2$, Figs. 2(b) and 2(c) show clearly that the $p-V$ data are only well described by the use of different parameter values for $V_{0}, K_{0}$, and $K_{0}^{\prime}$ as illustrated by the deviations of the data at higher pressures with respect to the (dashed) extrapolation for the $c F 4$ phase. In other words, new parameters at least for $V_{0}$ and $K_{0}$ must be adapted for each of the high-pressure phases.

Due to the fact that $V_{0}$ is known for the $c F 4$ (ambient pressure) phase one can try to fit even a second-order form with the two free parameters $K_{0}$ and $K_{0}^{\prime}$ to the data. For later comparison with $H 11$, the corresponding ${ }^{3,4,6,9}$ second-order form $H 12$

$$
\begin{aligned}
p= & 3 K_{0} X^{-5}(1-X) \exp \left[c_{0}(1-X)\right. \\
& \left.+\frac{3}{2} X\left(K_{0}^{\prime}-3-\frac{2 c_{0}}{3}\right)(1-X)\right],
\end{aligned}
$$

is used in this case. The resulting best fitted values for the $c F 4$ phase are given in the upper and middle part of Table I, the lower part of this table shows previous values obtained by the use of the Birch-Murnaghan-EOS form ${ }^{27}$ just with the latest data $\operatorname{set}^{12}$ in addition to values of $K_{0}$ and $K_{0}^{\prime}$ from
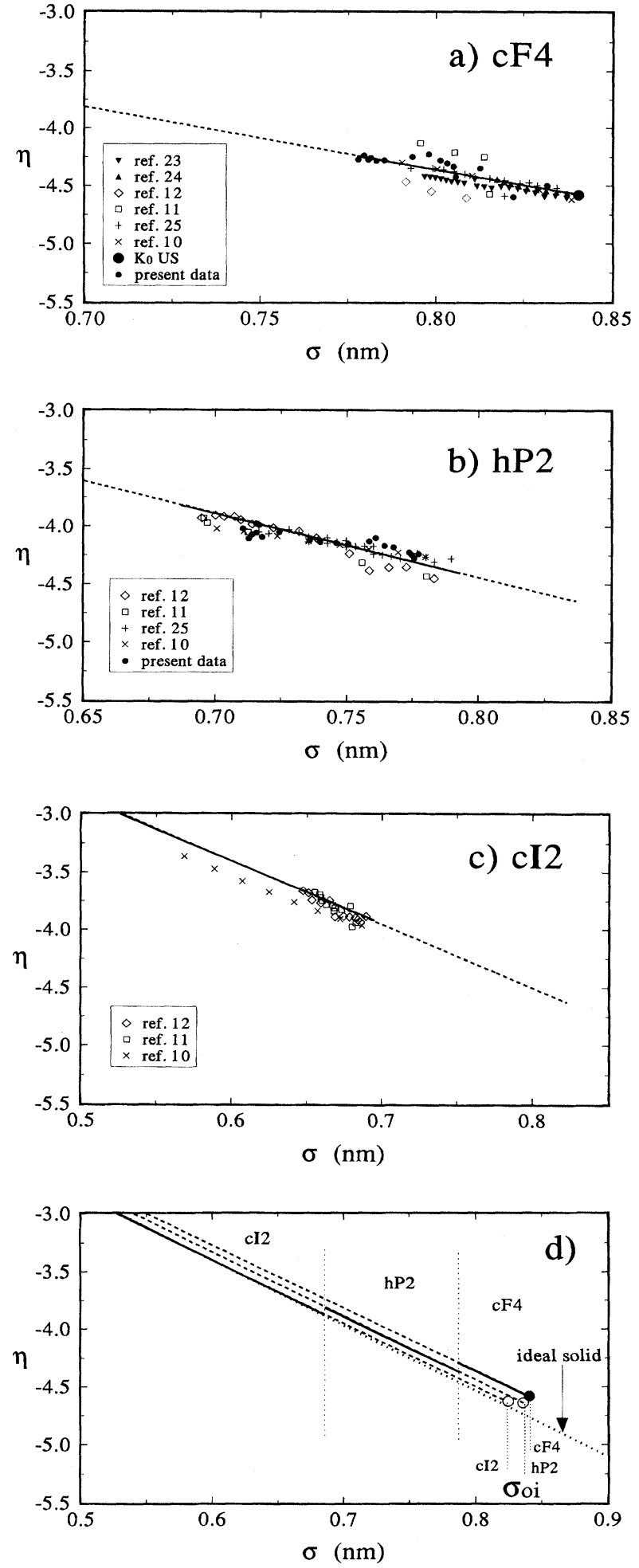

FIG. 2. Pressure-volume data of lead in $\eta$ - $\sigma$ representation for the individual phases [2(a)-2(c)]. The same form $H 11$ with different values for the parameters $V_{0 i}$ and $K_{0 i}$ fits all the data for the three phases and the differences in these fits are illustrated in (d) in comparison with the behavior of an "ideal" solid. 
TABLE I. Bulk modulus and its first pressure derivative at ambient condition for the low-pressure $c F 4$ phase. The upper part of this table shows the values of $K_{0}$ and $K_{0}^{\prime}$ obtained by fitting the form $H 12$ to the data. The middle part gives the parameters for the form $H 11$. The lower part reproduces values from the literature, whereby $\mathrm{BE}$ stands for the use of a Birch equation. $\mathrm{TH}$ designates theoretical data, and US stand for ultrasonic data.

\begin{tabular}{|c|c|c|c|c|}
\hline$K_{0}(\mathrm{GPa})$ & $K_{0}^{\prime}$ & $\sigma_{V}(\%)$ & Ref. & EOS \\
\hline $40(2)$ & $5(1)$ & 0.10 & 23 & \\
\hline $40(2)$ & $7(1)$ & 0.04 & 24 & \\
\hline $48(2)$ & $6.9(6)$ & 0.07 & 11 & \\
\hline $43.2(2)$ & $4.9(1)$ & 0.01 & 12 & \\
\hline $41(4)$ & $6(1)$ & 0.22 & 25 & $H 12$ \\
\hline $41(2)$ & $6.7(8)$ & 0.09 & 10 & \\
\hline $48(5)$ & $4(1)$ & 0.59 & This work & \\
\hline $42(5)$ & $5(1)$ & 0.43 & All data & \\
\hline $39(2)$ & 6.1 & 0.19 & 23 & \\
\hline $42(3)$ & 6.1 & 0.08 & 24 & \\
\hline $51(2)$ & 5.9 & 0.24 & 11 & \\
\hline $40(2)$ & 6.1 & 0.32 & 12 & \\
\hline $42(3)$ & 6.1 & 0.35 & 25 & $H 11$ \\
\hline $43(2)$ & 6.0 & 0.15 & 10 & \\
\hline $42(3)$ & 6.1 & 1.23 & This work & \\
\hline $40(4)$ & 6.1 & 0.85 & All data & \\
\hline 43.2 & 4.9 & & 12 & $\mathrm{BE} 2$ \\
\hline 46.2 & 4.6 & & 13 & $\mathrm{TH}$ \\
\hline 41.9 & 5.7 & & 26 & US \\
\hline
\end{tabular}

theoretical calculations ${ }^{13}$ and values derived from ultrasonic measurements. ${ }^{26}$ Obviously, the scatter of the experimental data for $K_{0}$ and $K_{0}^{\prime}$ in the first section of Table $\mathrm{I}$ is much larger than the purely statistical standard deviations of the fitted parameters given in brackets. Small standard deviations $\sigma_{V}$ of the measured volume data with respect to the fitted curves are noticed for the data ${ }^{23,24}$ which had been presented as smoothed compilations. If one takes all the data of the low-pressure phase together, one can notice that the values for both $K_{0}$ and $K_{0}^{\prime}$ show close agreement (within their statistical uncertainty) with the completely independent determination by ultrasonic measurements.

Furthermore, the second section of this table with the fitted parameter $K_{0}$ and the correlated parameter $K_{0}^{\prime}$ corresponding to the first-order form $H 11$ shows similarly good agreement with the ultrasonic data and only a minor increase in $\sigma_{V}$. Thus, the simple first-order form $H 11$ represents the best interpolation between low-pressure and ultimate compression for the given accuracy of the present data.

For the high-pressure phases, one has to consider $V_{0}$ also as free parameter in the fits, which increases the number of the free parameters in the common second-order forms to 3 and results in rather unstable minima of the fits due to the strong correlations of these parameters. ${ }^{6}$ However, perfect fits are obtained already with the first-order form $H 11$ and the corresponding results are represented in Table II for the intermediate $h P 2$ phase and in Table III for the highpressure $c I 2$ phase. In both tables, previous results ${ }^{12}$ based
TABLE II. Results of fitting the form $H 11$ to the data of the $h P 2$ phase. The lower part of this table reproduces values from the literature.

\begin{tabular}{lcccc}
\hline \hline$V_{0}\left(10^{-6} \mathrm{pm}^{3}\right)$ & $K_{0}(\mathrm{GPa})$ & $K_{0}^{\prime}$ & $\sigma_{V}(\%)$ & Ref. \\
\hline & $40(1)$ & 6.1 & 1.0 & 12 \\
& $37(1)$ & 6.2 & 1.0 & 11 \\
$30.0(5)$ & $41(3)$ & 6.1 & 0.6 & 25 \\
& $40(5)$ & 6.1 & 1.3 & 10 \\
& $41(4)$ & 6.1 & 1.6 & This work \\
& $40(2)$ & 6.0 & 2.0 & All data \\
29.98 & & & & \\
30.18 & 46.6 & 5.2 & & 12 \\
\hline \hline
\end{tabular}

on the Birch-Murnaghan-EOS form and the theoretical data ${ }^{13}$ are also included for comparison.

Due to the fact that the value for $K_{0}^{\prime}$ is directly coupled to the parameter $K_{0}$ in the first-order form $H 11$, no extra statistical uncertainties are given in these tables for the values of $K_{0}^{\prime}$. The comparison of the fits for the high-pressure phases with the results for the ambient pressure phase can be used, however, to estimate an uncertainty in the values of $K_{0}^{\prime}$ of typically $20 \%$. Within this uncertainty, "reasonable" agreement between the theoretical values ${ }^{13}$ and these best "experimental" data of $K_{0}^{\prime}$ for the high-pressure phases can then be noticed in Tables II and III. However, in the comparison of the experimental and theoretical values for $K_{0}$ of the high-pressure phases, one must take into account that the present lower values for $V_{0}$ of the high-pressure phases in comparison with extrapolation of the Birch-Murnaghan form $^{12}$ and the theoretical results lead automatically also to larger values for the corresponding parameters $K_{0}$.

Nevertheless, the best fitted straight lines in Fig. 2, which correspond to the first-order form $H 11$ with different parameter values for the individual phases, show now clear discontinuities with $\Delta V / V=1.7$ and $0.7 \%$ at the $c F 4 \rightarrow h P 2$ and $h P 2 \rightarrow c I 2$ phase transitions, respectively, in contrast to the smooth curves previously derived ${ }^{10,25}$ from shock-wave data.

Finally, if one compares the best-fitted EOS curves for the different phases of lead in Fig. 2 with the hypothetical curve of an ideal solid, which describes the average $p-V$ behavior of the most regular elements, ${ }^{4}$ one can notice that the data of lead are located only very slightly above this universal curve for ideal solids. The largest difference is noticed thereby for

TABLE III. Results of fitting the form $H 11$ to the data of the $c I 2$ phase. The lower part of this table reproduces the values from the literature.

\begin{tabular}{lcccc}
\hline \hline$V_{0}\left(10^{-6} \mathrm{pm}^{3}\right)$ & $K_{0}(\mathrm{GPa})$ & $K_{0}^{\prime}$ & $\sigma_{V}(\%)$ & Ref. \\
\hline \multirow{3}{*}{$28.5(5)$} & $51(1)$ & 6.0 & 0.9 & 12 \\
& $47(4)$ & 6.0 & 0.5 & 11 \\
& $41(5)$ & 6.1 & 0.6 & 10 \\
29.68 & $49(3)$ & 6.0 & 2.0 & All data \\
30.30 & & & & \\
\hline \hline
\end{tabular}


the low-pressure data and the differences become successively smaller for the high-pressure phases. This means that lead in its $c I 2$ phase behaves almost like an ideal solid, whereas the phases $h P 2$ and $c F 4$ show just the behavior of simple solids characterized by straight lines of the form $H 11$ but with slight deviations in their slopes with respect to the ideal case.

\section{ACKNOWLEDGMENTS}

Financial support by the Bundesminiterium für Wissenschaft und Forschung (BMFT) under Contract No. 055 PPAXB is gratefully acknowledged.
${ }^{1}$ A. L. Ruoff, H. Xia, and Q. Xia, Rev. Sci. Instrum. 63, 4342 (1992).

${ }^{2}$ L. Davidson and R. A. Graham, Phys. Rep. 55, 255 (1979).

${ }^{3}$ W. B. Holzapfel, in Molecular Systems under High Pressure, edited by R. Pucci and G. Piccitto (Elsevier Science, NorthHolland, Amsterdam, 1991), p. 61.

${ }^{4}$ W. B. Holzapfel, Europhys. Lett. 16, 67 (1991).

${ }^{5}$ O. Schulte and W. B. Holzapfel, Phys. Rev. B 48, 767 (1993).

${ }^{6}$ Y. K. Vohra and W. B. Holzapfel, High Pressure Res. 11, 223 (1993).

${ }^{7}$ R. G. Greene, H. Luo, and A. L. Ruoff, Phys. Rev. B 51, 597 (1995).

${ }^{8}$ W. A. Grosshans and W. B. Holzapfel, Phys. Rev. B 45, 5171 (1992).

${ }^{9}$ W. B. Holzapfel, J. Phys. Chem. Solids 55, 711 (1994).

${ }^{10}$ W. J. Nellis, J. A. Moriarty, A. C. Mitchell, M. Ross, R. G. Dandrea, N. N. Ashcroft, N. C. Holmes, and G. R. Grathers, Phys. Rev. Lett. 60, 1414 (1988).

${ }^{11}$ H. K. Mao, Y. Wu, J. F. Shu, J. Z. Hu, R. J. Hemley, and D. E. Cox, Solid State Commun. 74, 1027 (1990).

${ }^{12}$ Y. K. Vohra and A. L. Ruoff, Phys. Rev. B 42, 8651 (1990).

${ }^{13}$ A. M. Liu, A. Garcia, M. L. Cohen, B. K. Godwal, and R. Jeanloz, Phys. Rev. B 43, 1795 (1991).

${ }^{14}$ W. B. Holzapfel and W. May, Adv. Earth Planet. Sci. 12, 73 (1982).
${ }^{15}$ W. A. Grosshans, E. F. Düsing, and W. B. Holzapfel, High Temp. High Pressures 16, 539 (1984).

${ }^{16} \mathrm{~J}$. W. Otto (unpublished).

${ }^{17}$ K. Syassen and W. B. Holzapfel, Europhys. Conf. Abstr. 1A, 75 (1975).

${ }^{18}$ W. B. Holzapfel, in High Pressure Chemistry, edited by H. Kelm (Reidel, Boston, 1978).

${ }^{19}$ G. J. Piermarini, S. Block, J. D. Barnett, and R. A. Forman, J. Appl. Phys. 46, 2774 (1975).

${ }^{20}$ H. K. Mao, P. M. Bell, J. W. Shaner, and J. D. Steinberg, J. Appl. Phys. 49, 3276 (1978).

${ }^{21}$ International Union of Pure and Applied Chemistry, Nomenclature of Inorganic Chemistry, Recommendations 1990 (Blackwell Scientific, Oxford, 1990).

${ }^{22}$ J. Donohue, The Structure of the Elements (Wiley, New York, 1974).

${ }^{23}$ P. W. Bridgman, Phys. Rev. 60, 351 (1941).

${ }^{24}$ S. N. Vaidya and G. C. Kennedy, J. Phys. Chem. Solids 31, 2329 (1970).

${ }^{25}$ G. C. Kennedy and R. N. Keeler, in American Institute of Physics Handbook, 3rd ed. (McGraw-Hill, New York, 1972).

${ }^{26}$ R. A. Miller and D. E. Scheule, J. Phys. Chem. Solids 30, 589 (1969).

${ }^{27}$ F. Birch, Phys. Rev. 71, 809 (1947). 